\title{
From Mechanisms to Functions: The Role of Synchronization in the Intrahippocampal Circuits
}

\author{
Ivan Mysin $\mathrm{a}^{*}$, Liubov Shubina a \\ a Laboratory of Systemic organization of Neurons, Institute of Theoretical and Experimental \\ Biophysics of Russian Academy of Sciences, 3 Institutskaya Str., Pushchino, Moscow Region, \\ 142290 Russian Federation \\ ${ }^{*}$ Corresponding author: Ivan Mysin \\ Laboratory of Systemic Organization of Neurons, Institute of Theoretical and Experimental \\ Biophysics of Russian Academy of Sciences \\ 3 Institutskaya Str., Pushchino, Moscow Region, 142290 Russian Federation \\ Tel. and fax: +7-4967-330553 \\ E-mail: imysin@mail.ru
}

Abstract: The brain rhythms are essential for information processing in neuronal networks. Oscillations recorded in different brain regions can be synchronized and have a constant phase difference, i.e. be coherent. Coherence between local field potential (LFP) signals from different regions in the brain may be correlated with the performance of cognitive tasks, from which it is concluded that these regions of the brain are involved in the task performance together. In this review, we discuss why coherence occurs and how it is coupled to the information transfer between different regions of the hippocampal formation. Coherence in theta and gamma frequency ranges is described since these rhythms are most pronounced during the hippocampus-dependent attention and memory. We review in vivo studies of interactions between different regions of the hippocampal formation in theta and gamma frequency bands. The kay provisions of the review: 1) coherence emerges from synchronous postsynaptic currents in principal neurons, occurring as a result of synchronization of neuronal spike activity; 2) synchronization of neuronal spike patterns in two regions of the hippocampal formation can be realised through induction or resonance; 3) coherence at a specific time point reflects the transfer of information between regions of the hippocampal formation, in particular, gamma coherence reflects the coupling of active neuronal ensembles. Overall, coherence is not an epiphenomenon, but an important physiological process that has certain generation mechanisms and performs important functions in information processing and transmission across the brain regions.

Keywords: theta rhythm, gamma rhythm, coherence, local field potential, hippocampus

Abbreviations: CCK neurons - cholecystokinin-containing basket neurons; EC entorhinal cortex; LFP - local field potentials; MEC - medial entorhinal cortex; MEC L3 - 3rd layer of the medial entorhinal cortex; MS - medial septum, PV neurons - parvalbumincontaining basket neurons

\section{Introduction}

Performing cognitive tasks requires dynamic coordination of activity across large groups of neurons in different areas of the brain. Such coordination can be realized by synchronization of neuronal activity by oscillations. One of the most commonly used methods to study oscillations in the brain is the local field potentials (LFP) recording techniques. A 
large number of studies of the relationship between oscillations (rhythms) in the LFP and the performance of cognitive tasks are presented in the literature. Numerous studies have demonstrated that the coherence of oscillations between different areas of the brain is a marker of information transfer between these areas (Fries, 2005, 2015; Womelsdorf et al., 2007; Colgin et al., 2009; Carr et al., 2012; Yamamoto and Tonegawa, 2017; Nandi et al., 2019; Jiang et al., 2020). In this review, we focused on the mechanisms of coherence between LFP signals recorded in related subregions of the hippocampal formation and tried to explain the role of coherence in information processing. Two types of oscillations, theta and gamma rhythms, are mainly involved in the synchronization of neuronal activity in the hippocampus during active wakefulness (Buzsáki, 2002, 2006; Buzsáki and Moser, 2013; Colgin, 2016); therefore, we focused on these rhythms.

LFP are formed as a result of currents through the membranes of neurons (Buzsáki et al., 2012; Einevoll et al., 2013). Currents through the membranes of pyramidal neurons make a dominant contribution to LFP for two reasons. On the one hand, pyramidal neurons account for $80 \%$ to $91 \%$ of the entire neuronal population (Jinno and Kosaka, 2006; Bezaire and Soltesz, 2013). On the other hand, experimental estimates show that synaptic currents make the largest contribution to LFP in the frequency range up to $100 \mathrm{~Hz}$ (Einevoll et al., 2007, 2013; Pettersen et al., 2008; Buzsáki et al., 2012). Synaptic contacts of axons coming from other brain regions or from local interneurons are selectively located on the specific compartments of hippocampal pyramidal neurons (Klausberger et al., 2003; Klausberger, 2009; Somogyi et al., 2014). This, together with the ordered structure of the processes of pyramidal neurons, leads to the summation of synaptic currents in one direction between the hippocampal layers (Einevoll et al., 2013). In our further discourse, we will rely on the idea that only pyramidal neurons are involved in the formation of LFP in the frequency range up to $100 \mathrm{~Hz}$, i.e. in the frequency range of rhythms we are interested in.

Rhythms are formed as a result of the summation of potentials generated by a large number of pyramidal neurons. It follows that coherent oscillations in two brain regions are explained by the fact that pyramidal neurons in one region receive synchronous excitatory or inhibitory input with a constant time delay relative to pyramidal neurons in the other. Synchronous spike activity of a large number of neurons is of fundamental importance for the generation of rhythms and coherence.

Numerous theoretical studies show that neuronal networks can generate synchronous oscillatory activity in a wide range of neuronal parameters and connections in computational simulations (Borisyuk and Hoppensteadt, 1999; Durstewitz, 2009; Wang, 2010). The emergence of discharge synchronization has been shown both in networks of neurons described by very simple models (Hopfield and Herz, 1995; Brunel, 2000; Borisyuk, 2002; Brunel and Hansel, 2006) and in networks with very complex multi-compartment models of neurons and synapses (Schneider et al., 2012; Cutsuridis and Poirazi, 2015; Bezaire et al., 2016; Migliore et al., 2018; Ecker et al., 2020). Synchronization of neuronal activity may result from the interactions by chemical synapses, gap junctions, and/or receiving a common input. In the following sections, we will consider the mechanisms of synchronization and coherence during the generation of hippocampal gamma and theta rhythms in detail. Let us only note that neuronal networks' tendency to generate synchronous oscillations begets two mechanisms of coherence. The first mechanism consists in the synchronous activity induction from the neuronal network of one structure to another due to a strong input. The second mechanism proposes that synchronization of oscillations in two brain regions may occur due to resonance, even if the connections between those regions are weak. Both mechanisms result in a constant time between discharges of neurons in two connected regions. This in turn leads to the constant time between postsynaptic currents through the 
membrane of pyramidal neurons in neighbouring areas. Thus, oscillations in these areas have a constant phase difference, that is, they are coherent. Below we provide examples and discuss the role of each mechanism in creating coherence in the gamma and theta frequency range in detail.

\section{Mechanisms of synchronization in gamma-band}

In the hippocampal-entorhinal network, the gamma frequency band is often divided into slow (25-55 Hz, gammas), mid-frequency (55-80 Hz, gammam), and fast (80-120 Hz, gammaf) gamma oscillations (Belluscio et al., 2012; Schomburg et al., 2014). Some authors do not single out gammam, calling all gamma frequency band $>60 \mathrm{~Hz}$ "fast gamma oscillations" (Colgin et al., 2009). The division of the gamma oscillations into several frequency bands reflects differences in their LFP dynamics and behavioural correlates (Colgin et al., 2009; Colgin, 2015a; Fernández-Ruiz et al., 2017). In this review, we will stick to dividing gamma oscillations into three frequency bands.

The mechanisms of gammam and gammaf in the hippocampus are poorly understood. It has been shown that the occurrence of gammam in the CA1 field may be caused by the signals from the principal neurons of the 3rd layer of the medial entorhinal cortex (MEC L3) (Colgin et al., 2009; Fernández-Ruiz et al., 2017). In particular, high coherence is observed between the activity recorded in the CA1 hippocampal field and the MEC L3 (Colgin et al., 2009; Schomburg et al., 2014; Fernández-Ruiz et al., 2017; Yamamoto and Tonegawa, 2017). Moreover, the power of gammam is highest in the layer of the apical tufts of pyramidal neurons (stratum lacunosum-moleculare), the termination site for entorhinal afferents (Schomburg et al., 2014; Fernández-Ruiz et al., 2017). To the best of our knowledge, there is no data on the involvement of interneurons in gammam generation. Thus, gammam in the CA1 field is probably a consequence of excitatory postsynaptic currents generated by EC afferents and the mechanism of gammam coherence between CA1 and MEC is likely to be simple rhythm induction.

A fast gamma rhythm is recorded in all areas of the hippocampus (Bott et al., 2016; Lasztóczi and Klausberger, 2017; Trimper et al., 2017; Fernández-Ruiz et al., 2021). The maximum power of gammaf is most often observed in the CA1 pyramidal layer near the minimum of theta rhythm (Fernández-Ruiz et al., 2017; Lasztóczi and Klausberger, 2017), during the maximal activity of pyramidal neurons (Somogyi et al., 2014). These two facts lead to the idea that gammaf reflects the action potentials generation by the principal neurons. But this is only a hypothesis that requires confirmation. It is very difficult to assign the mechanisms of coherence between LFP signals from different hippocampal regions at frequencies above $100 \mathrm{~Hz}$, although gammaf coherence has been shown in some works (Fernández-Ruiz et al., 2021).

Research on gammas is more numerous. It is now believed that gammas is generated by parvalbumin-containing (PV) basket neurons (Colgin and Moser, 2010; Buzsáki and Wang, 2012). In our further reasoning, we will rely on the fact that it is precisely the inhibitory postsynaptic currents of synapses from the PV to the principal neurons generate gammas in LFP. Gammas synchronization of PV neurons is provided by the intrinsic properties of neurons and by the network mechanisms. PV neurons are practically devoid of adaptation currents (Buhl et al., 1994; Wang and Buzsáki, 1996), in addition, their bodies are relatively small, and as a result, the membrane has a low capacitance. These two properties result in PV neurons being able to discharge at a high frequency (Wang and Buzsáki, 1996). And the greatest resonance of their responses is observed in the gammas frequencies (Pike et al., 2000; Cardin et al., 2010). 
Two main mechanisms of PV neuronal spikes synchronization during gammas have been suggested. The first mechanism involves synchronization within the PV neuronal population through gap junctions and inhibitory synapses (Wang and Buzsáki, 1996; Saraga et al., 2006; Bartos et al., 2007). The second mechanism consists in the interaction between PV and principal neurons (Csicsvari et al., 2003; Colgin and Moser, 2010; Buzsáki and Wang, 2012). It has more evidence in the experimental data and is better explored in theoretical models (Börgers et al., 2012; Keeley et al., 2017; Butler et al., 2018). Nevertheless, both mechanisms do not contradict each other and most likely function simultaneously, which makes gammas one of the most common rhythms in the brain. Gammas oscillations have been observed in many brain regions, in a wide variety of physiological states and in the experiments in vitro (Traub et al., 1996; Schneider et al., 2015; Lundqvist et al., 2016; de la Prida and Huberfeld, 2019; Gilbert and Zarate, 2020). A high tendency of neuronal networks to generate gammas leads to the possibility of resonance between two connected brain structures, and as a result, to high coherence.

The described mechanism of gammas generation suggests a significant activity of the principal neurons. In freely behaving animals, the highest discharge frequency is manifested by neurons in the active neuronal ensemble, for example, the place encoding the current animal position (Burgess and O'Keefe, 2011; Jeffery, 2011). Hence gammas may coordinate the work of neuronal ensembles. It has been shown that discharges of pyramidal neurons are stronger modulated by gammas when the animal is in the centre of their place field (Senior et al., 2008). Other studies demonstrate that place cells discharging within the gamma cycle show a strong correlation with each other's discharge pattern, suggesting that they are part of the same neuronal ensemble (Harris et al., 2003). Based on these considerations, it can be assumed that high coherence between the two regions of the hippocampus coordinates the activity of neuronal ensembles in these regions and indicates the transfer of information between them. This idea finds a lot of experimental evidence. Studies in rodents (Montgomery and Buzsáki, 2007; Colgin et al., 2009; Carr et al., 2012; Chang and Huerta, 2012; Zheng et al., 2016; Fernández-Ruiz et al., 2017, 2021; Lasztóczi and Klausberger, 2017; Trimper et al., 2017; Yamamoto and Tonegawa, 2017), monkeys (Jutras et al., 2009; Montefusco-Siegmund et al., 2017), and humans (Fell et al., 2001, 2003b)_have shown that increased synchronization of hippocampal activity in the gamma frequency ranges correlates with the performance of various behavioural tasks, as well as with successful memory encoding and retrieval.

\section{Role of gamma coherence in navigation}

In freely moving animals (sometimes prompted to run by food or water reward), there is an interaction between different regions of the hippocampal formation in gamma frequency bands. Thus, in freely exploring rats during behavioural theta states, theta and gamma oscillations in the EC were coupled with theta and gamma oscillations in the dentate hilar region of the hippocampus (Chrobak and Buzsáki, 1998). Although more than 20 years have passed since this study, it is not entirely clear whether the communication between different regions of hippocampal formation is controlled by theta or gamma activity. Given that these two types of oscillations often coexist and are coupled (Bragin et al., 1995; Colgin et al., 2009; Belluscio et al., 2012), it is necessary to understand how they participate and interact in the activity coordination of different hippocampal subregions.

An important finding was that different types of gamma oscillations, gammas and gammam, were observed in the hippocampus while rats explored a familiar environment (theta activity state) and that they selectively synchronize CA1 with different afferent inputs. Gammas coupled CA1 to CA3, and gammam to MEC. A significant part of CA3 and MEC 
neurons were phase-locked to CA1 gammas and gammam, respectively. These two types of gamma oscillations occurred at different phases of the theta rhythm recorded in CA1 and often on different theta cycles (Colgin et al., 2009). If we assume that gamma synchronization facilitates communication between brain regions (Womelsdorf et al., 2007), it is likely that gammam promote transmission from MEC to CA1 and gammas underlie the transfer of information from CA3 to CA1.

Later, it was found that in the CA1 field of the hippocampus during theta states gammas prevailed in stratum radiatum, the termination site for CA3 inputs, while gammam was observed in the stratum lacunosum-moleculare, the termination site for EC. In addition, significant phase-locking was found between EC gammam LFP and unit spiking in CA1. The authors also believe that gammas modulated the activity of CA3 pyramidal cells entrains gammas in CA1 (Schomburg et al., 2014; Fernández-Ruiz et al., 2017).

Registration of neuronal activity from the CA1, CA3, dentate gyrus (DG), MEC and lateral EC (LEC) in rats during linear track running helped to confirm the assumption that the activity of CA1 pyramidal cells is tuned by the interaction of competing CA3 and EC inputs. The strength of these inputs reflects the power of gamma oscillations of a certain frequency band occurring in different phases of the theta rhythm (Fernández-Ruiz et al., 2017). Thus, the activity of CA1 place cells is initiated by the MEC L3 input at the peak of theta wave when the animal enters the place field and is associated with gammam in stratum lacunosummoleculare. As the animal runs through the place field, the influence of MEC decreases and the CA3 drive increases, as augment gammas in CA1 stratum radiatum (Fernández-Ruiz et al., 2017). In the CA3 field, in turn, gammas may originate from upstream DG microcircuits (Bragin et al., 1995; Hsiao et al., 2016): DG gammas influence CA3 gammas and spike activity of DG place cells is synchronized and phase-locked to CA3 gammas (Bragin et al., 1995; Hsiao et al., 2016).

It is interesting to note that gammas and gammam in the CA1, CA3 and EC were shown to change differently depending on the running speed of the animal (Zheng et al., 2015). With an increase in running speed, the power and frequency of gammam increased in all three areas, while gammas were significantly less dependent on speed. Firing rates of CA3 neurons were low at high running speed, whereas MEC firing rates were high. This allows to suggest that gammam is triggered by MEC at high running speeds and gammas is mediated by CA3 at low running speeds (Zheng et al., 2015). These results again support the hypothesis that gammas and gammam likely present functionally different states in hippocampal formation. In another study, the effect of the animal running speed was investigated on the relationship between CA1 and DG. Within these regions, an increase in the running speed also led to an increase in the power and phase-locking between theta and gamma oscillations. At moderate running speeds, a significant coupling was found between DG and CA1 gamma, although it was shown that CA1 gamma oscillations were mainly driven by the local theta rhythm (Sheremet et al., 2020).

A study by Lasztóczi and Klausberger (Lasztóczi and Klausberger, 2017) in awake head-fixed mice also report a significant phase coherence between DG and CA1 (str. radiatum and str. pyramidale) at gammas during theta states. Furthermore, DG and CA1 gammas modulated the spike activity in the DG and CA1 (although to a lesser extent). All this may indicate that DG and CA1 gammas are not independent (Lasztóczi and Klausberger, 2017) and rather reflect the same basic oscillatory process that regulates information processing in the entire hippocampal formation, and possibly beyond it. On the contrary, DG gammaf $(75-150 \mathrm{~Hz})$ and CA1 gammaf $(92-150 \mathrm{~Hz})$ were rather independent processes, demonstrating low phase coherence and CA1 pyramidal layer unit firing independence of DG gammaf (although weak reverse modulation of DG units by CA1 gammaf was noticed) 
(Lasztóczi and Klausberger, 2017). Activity in different layers of the CA1 and DG was studied in rats during such a marker of the exploratory behaviour as rearing. During rearing an increase in the frequency of theta oscillations was observed in all layers of CA1, as well as coupling of CA1 theta rhythm to the amplitude of DG gammaf $(120-150 \mathrm{~Hz})$. Additional analysis indicated that during rearing signalling through the perforant pathway (through DG from EC layer 2) increased and the MEC L3 input to the CA1 was weakening (Barth et al., 2018).

A recent study under similar conditions (theta states during free behaviour) in rats found that DG gammam amplitude was significantly coupled to the CA1 theta phase, but not vice versa. Similarly, the amplitude of hippocampal gammam was coupled to the theta phase in the prefrontal cortex (Nandi et al., 2019), which corresponds to the direction of the anatomical connections between these regions. The authors of this study believe that the analysis of the phase-amplitude relationship between different areas of the brain can be used to determine the relationship between the output activity of a rhythmic "driver" (gammam) network and the input activity of a rhythmic "receiver" network (theta), thereby establishing the direction and strength of information transmission (Nandi et al., 2019). It should be noted that both in the CA1 and DG, regardless of the behaviour of the animals (for example, running or REM sleep), the theta and gamma coherence decrease across the septo-temporal (long) axis of the hippocampus, and this decline is faster for gamma frequencies (Penley et al., 2012).

Also confirming the directionality of the anatomical relationships, another study showed that the directions of theta and gamma cross- and within-frequency interactions were always from the CA3 to CA1 field (Jiang et al., 2020). LFP recordings from these areas in freely behaving rats allowed to demonstrate that CA3 gammas power adjusted the CA1 theta phase, suggesting that CA3 gammas entrain CA1 theta oscillations (Jiang et al., 2020).

The rhythmical activity of hippocampal neurons in freely moving animals demonstrates characteristic experience-dependent changes, namely, it differs in early and late sessions of animal navigation in a familiar environment. In earlier sessions, when rats run along a linear track for a reward, CA3 pyramidal neurons spike activity was lower, while that of EC neurons and neurons in the deep layers of CA1 (EC efferents) was higher. CA1 neuronal spikes were more strongly modulated by gammam and weaker by gammas. This, in general, probably indicates a stronger MEC L3 influence on the CA1 activity in the first 4 sessions, which decreases with time allowing the CA3 input to gain control (Fernández-Ruiz et al., 2017) . The influence of the experience was also observed in the LFP of the CA1 pyramidal layer. In the first minutes of the first session of the animals' traversing a familiar circular track with randomly located food rewards higher increase in fast theta and a decrease in gammas were seen. The gammam power gradually decreased in each session, regardless of time, indicating less influence of the experience on this frequency range (Gereke et al., 2018) . The authors hypothesize that experience-dependent changes in hippocampal rhythms may be associated with changes in the activity of place cells, appearing with learning.

\section{Role of gamma coherence in learning and memory}

The role of synchronization in gamma frequency bands across hippocampal formation has also been investigated in various spatial memory and learning tasks. It has been shown that different types of gamma oscillations can be involved both in encoding (Fell et al., 2003a; Jutras et al., 2009; Tort et al., 2009; Fell and Axmacher, 2011) and retrieval (Montgomery and Buzsáki, 2007; Schomburg et al., 2014; Fernández-Ruiz et al., 2017; Yamamoto and Tonegawa, 2017) of spatial memory and increased synchronization of gamma oscillations between different areas may be a marker of better memory. 
Simultaneously recording of CA1, CA3 and DG LFP from rats during the performance of a hippocampus-dependent delayed spatial alternation task on a modified T-maze Montgomery and Buzsáki in 2007 found that gamma coherence between the CA3 and CA1 fields was enhanced. Importantly, gamma synchronization increased at the moment of memory-based decision-making (before the T junction) (Montgomery and Buzsáki, 2007). Later, it was shown that at such a moment (memory recall) the CA3-CA1 coupling increased at the frequency of gammas (Schomburg et al., 2014). During the recall phase (central arm), the firing rates of CA3 pyramidal cells and CA1 interneurons were significantly higher compared to the lateral arms of the maze, where increased excitation of MEC neurons and CA1 gammam power in str. lacunosum-moleculare were observed (Schomburg et al., 2014). These results were confirmed in the further work of this group (Fernández-Ruiz et al., 2017), where the activity of neurons in hippocampal formation during the performance of a spatial alternation task on a modified T-maze was studied in more detail. Among other things, it was shown that in the central arm of the maze (memory-based decision-making point), the CA1 spiking activity was more strongly modulated by CA3 gammas, compared to the lateral arms. If the wrong decision was made, no difference in neuronal activity was found between the central and lateral arms. In addition, according to the authors' hypothesis, stronger excitation of CA1 neurons by CA3 neurons in the central arm during memory recall led to reduction of phase precession (Fernández-Ruiz et al., 2017).

Memory reactivation associated increase in gammas power and gammas synchronization between the CA3 and CA1 fields was also observed during sharp-wave ripple events in another hippocampus-dependent spatial alternation task (change in the position of the W-track), where higher levels of gamma synchrony correlated with better memory (Carr et al., 2012). In contrast to the CA3 and CA1, successful performance of the delayed spatial alternation task (correct arm choice in T-maze) was preceded by an increase in gammaf synchronization between the MEC and CA1 (Yamamoto and Tonegawa, 2017). Thus, gamma synchrony across the hippocampal formation plays probably a central role in the coordination of memory reactivation, maintaining the temporal organization of neuronal ensembles.

Gamma synchronization is also involved in memory encoding processes. Thus, a study in humans (epileptic patients) related successful performance of memorization tasks to increased gamma-band (about $40 \mathrm{~Hz}$ ) LFP synchronization (followed by desynchronization) between the hippocampus and perirhinal/entorhinal cortex during memory formation (Fell et al., 2001). Then it was shown that also theta coherence increased during successful recognition memory encoding (Fell et al., 2003b). Further, a study in monkeys extended these observations to hippocampal neurons, demonstrating that they are synchronized in the gammam (> $60 \mathrm{~Hz}$ ) during memory formation (successful encoding of novel images). Hippocampal spikes were phase-locked among each other and with the LFP gammam. Moreover, the magnitude of synchronization was correlated with the success of subsequent memory performance (recognition of new and repeated pictures) (Jutras et al., 2009).

During learning, the manifestation of gamma activity and its coupling to theta rhythm are also changed. In the CA3 pyramidal layer of the hippocampus theta-gamma coupling continuously increased (theta phase-gamma amplitude coupling) as the animals learned to associate objects with the context. The task performance accuracy significantly correlated with the strength of this coupling (Tort et al., 2009). An increase in the theta-gamma coupling during the learning process was also shown for the CA1 in spatial memory tasks. As the training session progressed, enhancement of theta-gamma coupling, as well as gammam power, along with a decrease in gammas power, were observed before the animal had to make a choice based on previous experience (Nishida et al., 2014). However, in another 
study using a modified Barnes maze a decrease in theta and gammam power as well as their coupling was found in the DG during learning. However, a learning-dependent increase in theta-gammam coupling was shown in the DG but not in the CA1 at the vicinity of the target area in the maze (Bott et al., 2016). These results conclusively demonstrate the functional role of hippocampal theta-gamma coupling in learning, which may be a mechanism of relevant information computation by different hippocampal regions.

In conclusion, the reviewed data show that gamma coherence between different parts of the hippocampal formation is not an epiphenomenon, but a process that reflects the interaction of active neuronal ensembles involved in the generation of complexity and flexibility of animal cognition and behaviour.

\section{Role of gamma coherence in novelty detection}

A change in gamma coherence between different regions of the hippocampal formation has been shown in novel object and novel object-place recognition tasks in a familiar and novel environment (hippocampus-dependent spatial associative memory).

Chang and Huerta (Chang and Huerta, 2012) demonstrated in mice, that when animals explored the environment (context), but not the new objects, there was an increase in gamma and theta coherence between the dorsal subiculum and CA1 and within these structures. When animals explored novel objects, the coherence between the dorsal subiculum and CA1 was low.

Probably, during contextual examination (when the animal navigates in the environment), subiculum and CA1 participate in spatial information encoding, leading to high subiculum-CA1 gamma and theta coherence. Conversely, during novel object exploration, subiculum encodes recognition signals, probably coupling with the perirhinal and postrhinal networks and reducing the connection with CA1, leading to low subiculum-CA1 gamma and theta coherence. An increase in the subiculum-CA1 coherence during navigation in the experimental field (although mainly in the theta frequency range) was also noted in another study in rats (Trimper et al., 2017).

It is important to note that novel object exploration also leads to an increase in gamma coherence, but between other regions of the hippocampal formation. It was found that when animals were exploring novel objects (recognition memory task) CA3-CA1 coherence increased markedly in gammas range and the degree of this gamma synchrony correlated with the subsequent memory performance (Trimper et al., 2014). In a subsequent study, it was shown that during novel object exploration, gammas coherence increased not only between the CA3 and CA1 fields but also between the DG and CA3 and it was also related with a good subsequent memory of both the object and its location, correlating with the degree of their novelty (Trimper et al., 2017). Thus, the degree of gamma synchronization in the hippocampus during novel object exploration correlates with the memory encoding for these objects. Gammas coherence increase may reflect the memory for an object encountered in a particular location, given that CA3 is thought to play an important role in memory retrieval (Sutherland et al., 1983; Brun et al., 2002; Steffenach et al., 2002). It can be assumed that gammas are probably predominant in the hippocampus during associative memory encoding.

Increased gammas phase-locking was also shown for CA1 place cell spikes in rats exploring a novel environment for the first time (Kitanishi et al., 2015). However, despite the probable relationship between the CA3 and CA1 fields during the arrival of novel information, in the study of Larkin and colleagues an increase in the activity of place cells when rats were exploring a novel object in a new place or an old object in a new place was detected only in the CA1, but not in the CA3 (Larkin et al., 2014). 
An increase in CA3-CA1 coherence in gammas range was also observed upon electrical stimulation of the basolateral nucleus of the amygdala immediately after the exploration of a novel object. This intervention resulted in better memory for these objects the day after stimulation (Bass and Manns, 2015). This study shows that CA3-CA1 activity synchronization in gammas range may underlie the amygdala-triggered neuronal network state that "selects" recently encoded information for long-term storage.

Another study found that novelty exploration increased gammam phase synchrony between CA3 and CA1 (Zheng et al., 2016). Such a reaction was observed when rats were exploring novel objects in a new place (where there were no objects before) in a familiar environment. According to this data, associative memory encoding of novel object-place is facilitated by gammam frequency range (Zheng et al., 2016). It is interesting to note that an increase in gammam coherence across the septo-temporal (long) axis of the CA1, as well as an increase in DG and CA1 gammam power, can be caused by just environmental novelty (Penley et al., 2013).

More recently, the different roles of gammas and gammaf in "spatial" and "object" learning tasks have been investigated. Fernández-Ruiz and colleagues examined gamma and spike activity coupling between the EC and DG during learning and after selective optogenetic disturbance of gamma synchronization (Fernández-Ruiz et al., 2021). During spatial learning, gammaf synchronization between MEC and DG was observed, while during object learning, gammas synchronized DG and LEC. Selective gamma-band perturbation of MEC or LEC reduced DG gammaf or gammas, respectively, and led to the impairment of spatial (MEC perturbation) or object (LEC perturbation) learning (Fernández-Ruiz et al., 2021). In general, depending on the tasks, specific patterns of gamma oscillations dynamically engage functional neuronal ensembles across different regions of the brain.

\section{Mechanisms of Synchronization in Theta-Band}

Hippocampal theta rhythm is necessary for the processes of attention and memory (Vinogradova, 1995, 2001; Buzsáki, 2002; Buzsáki and Moser, 2013). In freely behaving animals theta rhythm in hippocampal formation appears as a result of rhythmic input from the medial septal area (MS) (Petsche and Stumpf, 1960; Stewart and Fox, 1990; Vertes and Kocsis, 1997; Brazhnik, 2004). Due to their local interactions, MS neurons generate rhythmic activity at the theta frequency (Ujfalussy and Kiss, 2006; Dannenberg et al., 2015; Mysin et al., 2015; Robinson et al., 2016). The main role in the transmission of rhythmic activity is played by two GABAergic neuronal populations of MS, producing in antiphase bursts of spikes at theta frequency during theta rhythm generation (Borhegyi et al., 2004; Hangya et al., 2009). The neurons of these populations were named Teevra and Komal cells, respectively, due to peculiarities of their spike train dynamics (Joshi et al., 2017). It is well known that GABAergic MS neurons selectively innervate interneurons in the hippocampus and EC (Freund and Antal, 1988; Freund, 1989; Gonzalez-Sulser et al., 2014). However, recent studies have shown that innervation of different areas of the hippocampus by MS projections is not equal. Teevra cells inhibit cholecystokinin-containing (CCK) basket and axo-axonal neurons in the CA3 field and, to a much lesser extent, in the CA1 field (Joshi et al., 2017). Komal cells send projections to MEC L3 and L2 interneurons and the subiculum (Viney et al., 2018). DG receives input mainly from low-rhythmic MS GABAergic cells (Salib et al., 2019). These MS neurons, in contrast to Teevra and Komal cells, do not express PV, more often fire by single spikes and weaker coupled to the hippocampal theta rhythm (Salib et al., 2019). LEC receives only cholinergic input from the MS (Desikan et al., 2018). It was shown that direct MS cholinergic projections do not induce theta rhythm in the hippocampus (Dannenberg et al., 2015), but theta rhythm in the LEC is well expressed, although its power 
is lower than in the MEC. Perhaps the reason is the prevalence of nicotinic receptors in the LEC compared to the prevalence of muscarinic receptors in the hippocampus and MEC (Desikan et al., 2018).

Data on the structure of septal projections show that the neuronal mechanisms of theta rhythm in different areas of hippocampal formation may vary. However, all regions of the hippocampal formation exhibit high power of the theta rhythm and high pairwise coherence (Kocsis et al., 1999; Mizuseki et al., 2009; Montgomery et al., 2009). This suggests the idea that different hippocampal regions can resonate at the theta frequency and contribute to the rhythmicity of each other. In addition to the network mechanisms, the hippocampal networks tendency to oscillate at the theta frequency is provided by the properties of pyramidal neurons, which have maximal responses at theta frequency stimulation (Pike et al., 2000; Wang et al., 2006; Cardin et al., 2009).

\section{Functional role of theta coherence}

The hypothesis that theta rhythm may synchronize neuronal populations to facilitate information transfer within and across neuronal ensembles (Fries, 2005; Sirota et al., 2008) is not new. In addition to the high theta coherence across the hippocampal formation (Kocsis et al., 1999; Mizuseki et al., 2009), it is known that activity of different neuronal populations to a greater or lesser extent are coupled to the phase of hippocampal theta rhythm (Klausberger et al., 2003; Mizuseki et al., 2009; Somogyi et al., 2014). It has even been demonstrated that theta activity can propagate along the septo-temporal axis of the hippocampus in a "travelling wave" (Petsche and Stumpf, 1960; Lubenov and Siapas, 2009; Patel et al., 2012).

Synchronization of oscillatory activity in other circuits with hippocampal theta rhythm during cognitive processes was also observed for many subcortical and cortical networks, such as the prefrontal cortex (Adhikari et al., 2010; Benchenane et al., 2010; Nandi et al., 2019), amygdala (Seidenbecher et al., 2003), striatum (DeCoteau et al., 2007), medial septum (Dragoi et al., 1999; Hernández-Pérez et al., 2015), and supramammillary nucleus (Kocsis and Vertes, 1994; Hernández-Pérez et al., 2015).

Nevertheless, despite the long history of investigation of theta rhythm and its functional role, in vivo studies on the interaction of hippocampal regions during theta generation are few and rather contradictory. Thus, it was shown that the dynamics of intrahippocampal communication may be controlled not only by the classically described unidirectional connections (Jackson et al., 2014). Recording LFP from the CA3, CA1, and subiculum during different animal behaviour, Jackson and colleagues found that theta rhythm in the subiculum could synchronize activity in the CA3 field. Optogenetic inhibition of interneurons projecting from the subiculum to the CA3 decreased theta power in the latter. We believe that these results require additional verification since they contradict the aforementioned data that CA3 receives the strongest input from the MS. However, if the results of Jackson et al. study are confirmed, it can be assumed that theta coherence may result from the resonance of several regions of the hippocampal formation in a closed circuit with feedback projections.

Several studies using surgical removal of the EC have demonstrated the role of entorhinal input through the perforating pathway in the generation of theta rhythm in the CA1 (Buzsáki et al., 1983; Montoya and Sainsbury, 1985). There is also evidence that synchronous theta spiking of EC stellate cells during EC theta oscillations is capable of driving induction of CA1 LTP via the trisynaptic hippocampal pathway (Stepan et al., 2012). Moreover, in vivo experiments confirm that theta oscillations in EC are closely related to theta spike activity in DG granular cells (Jung and McNaughton, 1993; Skaggs et al., 1996; Mizuseki et al., 2009). 
On the other hand, in a recent study using optogenetics, it was shown that EC neuronal activity blockade did not change CA1 theta power. In the same study, it was demonstrated that blocking the Schaffer's collaterals input from CA3 to CA1 decreased the CA1 theta power (López-Madrona et al., 2020). However, in an earlier study, it was noted that during the Schaffer's collaterals blockade the decrease in the CA1 theta power was statistically insignificant (Middleton and McHugh, 2016). We would like to note that in the study by Middleton and McHugh, a gene modification technique was used, in which the blockade of synapses from the CA3 pyramidal neurons appeared within two weeks. We believe data obtained with the help of optogenetics is more reliable since in this case the effect is acute. In addition, the idea of the CA3 input influence on the CA1 theta rhythm is supported by the already mentioned data on the Granger causality between the CA3 and CA1 LFP recordings (Jiang et al., 2020).

There is an assumption that at different phases of the CA1 theta rhythm, different functions are performed (Hasselmo et al., 2002; Manns et al., 2007; Cutsuridis et al., 2010; Cutsuridis and Poirazi, 2015; Fernández-Ruiz et al., 2017). This hypothesis is based on the fact that the CA1 receives two information inputs: from CA3 through the Schaffer's collaterals and from the EC through the perforant pathway. The signal from these two inputs arrives at different phases of the theta rhythm (Mizuseki et al., 2009; Montgomery et al., 2009). The authors suggest that at the minimum of theta wave, the largest input comes from the EC, and the CA1 neuronal network operates in the "recording" mode. At the theta wave peak, the largest input comes from CA3, and CA1 reproduces the stored information. It should be noted that the principal neurons of the MEC L3 fire at the peak of the theta wave in the CA1 pyramidal layer; however, the signal is transmitted with a long delay of $70-80 \mathrm{~ms}$ and arrives near the minimum of CA1 theta (Mizuseki et al., 2009).

Another aspect of theta coherence is synchronization between the dorsal and ventral hippocampus. Their activities are coherent and phase difference increases across the septotemporal axis of the hippocampus. In other words, the theta rhythm propagates as a travelling wave from the dorsal to the ventral pole of the hippocampus (Lubenov and Siapas, 2009; Patel et al., 2012; Zhang and Jacobs, 2015). The mechanisms of such theta rhythm propagation are currently unknown. Three hypotheses are widely discussed (Lubenov and Siapas, 2009; Patel et al., 2012; Zhang and Jacobs, 2015).

The first hypothesis implies the delay in the arrival of the signal from the MS in the ventral hippocampus relative to the dorsal one. The second hypothesis is that the inputs from the MS induce the rhythmical activity in the dorsal hippocampal neurons, which, in turn, impose rhythmical activity onto neighbouring neurons, which transmit it further etc. The third hypothesis, similarly to the second, suggests that dorsal hippocampal neurons are entrained into rhythmic activity by projections from MS; however, the propagation of the theta rhythm across the hippocampus occurs due to resonance between the oscillatory activity of pyramidal neurons (Pike et al., 2000; Wang et al., 2006; Cardin et al., 2009). We believe that the third mechanism is the most probable. Although studies show that MS innervates the dorsal and ventral hippocampus to the same extent, the MS projections are heterogeneous. The rostral part of the MC predominantly innervates the dorsal, and the caudal part of the MC predominantly innervates the ventral hippocampus (Yoshida and Oka, 1995; Ohara et al., 2013). The theta rhythm parameters in the dorsal and ventral hippocampus are significantly different. Theta power is higher in the dorsal hippocampus than in the ventral (Royer et al., 2010). Theta rhythm modulation of pyramidal cell and interneuron spikes is also significantly lower in the ventral hippocampus (Royer et al., 2010). In addition, indirect evidence of the hypothesis that theta rhythm propagates from the dorsal to the ventral pole of the hippocampus by the resonance mechanism is provided by data on the activity of 
principal neurons. During theta rhythm, hippocampal principal neurons rarely fire by single spikes (Soltesz and Deschênes, 1993; Ylinen et al., 1995; Csicsvari et al., 1998). According to experimental data, no more than $1 \%$ of principal neurons in all regions of the hippocampus discharges in one theta cycle, while almost all principal neurons exhibit subthreshold oscillations of the membrane potential at theta frequency (Soltesz and Deschênes, 1993; Ylinen et al., 1995; Csicsvari et al., 1998). Thus, almost all hippocampal neurons are involved in the generation of theta rhythm.

\section{Functional role of theta coherence}

The hypothesis that theta rhythm may synchronize neuronal populations to facilitate information transfer within and across neuronal ensembles (Fries, 2005; Sirota et al., 2008) is not new. In addition to the high theta coherence across the hippocampal formation (Kocsis et al., 1999; Mizuseki et al., 2009), it is known that activity of different neuronal populations to a greater or lesser extent are coupled to the phase of hippocampal theta rhythm (Klausberger et al., 2003; Mizuseki et al., 2009; Somogyi et al., 2014). It has even been demonstrated that theta activity can propagate along the septo-temporal axis of the hippocampus in a "travelling wave" (Petsche and Stumpf, 1960; Lubenov and Siapas, 2009; Patel et al., 2012).

Synchronization of oscillatory activity in other circuits with hippocampal theta rhythm during cognitive processes was also observed for many subcortical and cortical networks, such as the prefrontal cortex (Adhikari et al., 2010; Benchenane et al., 2010; Nandi et al., 2019), amygdala (Seidenbecher et al., 2003), striatum (DeCoteau et al., 2007), medial septum (Dragoi et al., 1999; Hernández-Pérez et al., 2015), and supramammillary nucleus (Kocsis and Vertes, 1994; Hernández-Pérez et al., 2015).

Nevertheless, despite the long history of investigation of theta rhythm and its functional role, in vivo studies on the interaction of hippocampal regions during theta generation are few and rather contradictory. Thus, it was shown that the dynamics of intrahippocampal communication may be controlled not only by the classically described unidirectional connections (Jackson et al., 2014). Recording LFP from the CA3, CA1, and subiculum during different animal behaviour, Jackson and colleagues found that theta rhythm in the subiculum could synchronize activity in the CA3 field. Optogenetic inhibition of interneurons projecting from the subiculum to the CA3 decreased theta power in the latter. We believe that these results require additional verification since they contradict the aforementioned data that CA3 receives the strongest input from the MS. However, if the results of Jacksons et al. study are confirmed, it can be assumed that theta coherence may result from the resonance of several regions of the hippocampal formation in a closed circuit with feedback projections.

Several studies using surgical removal of the EC have demonstrated the role of entorhinal input through the perforating pathway in the generation of theta rhythm in the CA1 (Buzsáki et al., 1983; Montoya and Sainsbury, 1985). There is also evidence that synchronous theta spiking of EC stellate cells during EC theta oscillations is capable to drive induction of CA1 LTP via the trisynaptic hippocampal pathway (Stepan et al., 2012). Moreover, in vivo experiments confirm that theta oscillations in EC are closely related to theta spike activity in DG granular cells (Jung and McNaughton, 1993; Skaggs et al., 1996; Mizuseki et al., 2009).

On the other hand, in a recent study using optogenetics, it was shown that EC neuronal activity blockade did not change CA1 theta power. In the same study, it was demonstrated that blocking the Schaffer's collaterals input from CA3 to CA1 decreased the CA1 theta power (López-Madrona et al., 2020). However, in an earlier study, it was noted that during the Schaffer's collaterals blockade the decrease in the CA1 theta power was statistically 
insignificant (Middleton and McHugh, 2016). We would like to note that in the study by Middleton and McHugh, a gene modification technique was used, in which the blockade of synapses from the CA3 pyramidal neurons appeared within two weeks. We believe data obtained with the help of optogenetics is more reliable since in this case the effect is acute. In addition, the idea of the CA3 input influence on the CA1 theta rhythm is supported by the already mentioned data on the Granger causality between the CA3 and CA1 LFP recordings (Jiang et al., 2020).

There is an assumption that at different phases of the CA1 theta rhythm, different functions are performed (Hasselmo et al., 2002; Manns et al., 2007; Cutsuridis et al., 2010; Cutsuridis and Poirazi, 2015; Fernández-Ruiz et al., 2017). This hypothesis is based on the fact that the CA1 receives two information inputs: from CA3 through the Schaffer's collaterals and from the EC through the perforant pathway. The signal from these two inputs arrives at different phases of the theta rhythm (Mizuseki et al., 2009; Montgomery et al., 2009). The authors suggest that at the minimum of theta wave, the largest input comes from the EC, and the CA1 neuronal network operates in the "recording" mode. At the theta wave peak, the largest input comes from CA3, and CA1 reproduces the stored information. It should be noted that the principal neurons of the MEC L3 fire at the peak of the theta wave in the CA1 pyramidal layer; however, the signal is transmitted with a long delay of $70-80 \mathrm{~ms}$ and arrives near the minimum of CA1 theta (Mizuseki et al., 2009).

Another aspect of theta coherence is synchronization between the dorsal and ventral hippocampus. Their activities are coherent and phase difference increases across the septotemporal axis of the hippocampus. In other words, the theta rhythm propagates as a travelling wave from the dorsal to the ventral pole of the hippocampus (Lubenov and Siapas, 2009; Patel et al., 2012; Zhang and Jacobs, 2015). The mechanisms of such theta rhythm propagation are currently unknown. Three hypotheses are widely discussed (Lubenov and Siapas, 2009; Patel et al., 2012; Zhang and Jacobs, 2015).

The first hypothesis implies the delay in the arrival of the signal from the MS in the ventral hippocampus relative to the dorsal one. The second hypothesis is that the inputs from the MS induce the rhythmical activity in the dorsal hippocampal neurons, which, in turn, impose rhythmical activity onto neighbouring neurons, which transmit it further etc. The third hypothesis, similarly to the second, suggests that dorsal hippocampal neurons are entrained into rhythmic activity by projections from MS; however, the propagation of the theta rhythm across the hippocampus occurs due to resonance between the oscillatory activity of pyramidal neurons (Pike et al., 2000; Wang et al., 2006; Cardin et al., 2009). We believe that the third mechanism is the most probable. Although studies show that MS innervates the dorsal and ventral hippocampus to the same extent, the MS projections are heterogeneous. The rostral part of the MC predominantly innervates the dorsal, and the caudal part of the MC predominantly innervates the ventral hippocampus (Yoshida and Oka, 1995; Ohara et al., 2013). The theta rhythm parameters in the dorsal and ventral hippocampus are significantly different. Theta power is higher in the dorsal hippocampus than in the ventral (Royer et al., 2010). Theta rhythm modulation of pyramidal cell and interneuron spikes is also significantly lower in the ventral hippocampus (Royer et al., 2010). In addition, indirect evidence of the hypothesis that theta rhythm propagates from the dorsal to the ventral pole of the hippocampus by the resonance mechanism is provided by data on the activity of principal neurons. During theta rhythm, hippocampal principal neurons rarely fire by single spikes (Soltesz and Deschênes, 1993; Ylinen et al., 1995; Csicsvari et al., 1998). According to experimental data, no more than $1 \%$ of principal neurons in all regions of the hippocampus discharges in one theta cycle, while almost all principal neurons exhibit subthreshold oscillations of the membrane potential at theta frequency (Soltesz and Deschênes, 1993; 
Ylinen et al., 1995; Csicsvari et al., 1998). Thus, almost all hippocampal neurons are involved in the generation of theta rhythm.

There is not a lot of information about the role of theta synchronization between different regions of the hippocampal formation in cognitive tasks. It is supposed that theta coherence is involved in the encoding and retrieval of episodic memory (Buzsáki, 1996; Hasselmo et al., 2002; Hasselmo, 2005). During the performance of the hippocampus-dependent spatial alternation task, it was found that, although theta oscillations were generally coherent throughout the hippocampus (CA1, CA3, and DG), the power, coherence, and phase of theta activity varied depending on the layer of registration and animal position in the maze, suggesting, as the authors thought, the presence of different theta generators. The phase delay between CA3 and CA1 theta in pyramidal layers was significantly smaller before the T-junction (Montgomery et al., 2009).

Similar to gamma oscillations (Montgomery and Buzsáki, 2007), the theta power and coherence increased in the central arm of the modified T-maze in a layer-specific way, regardless of the running speed or acceleration of the animal. However, in contrast to gamma oscillations, theta power and coherence also increased in the initial segment (when the animal entered the maze) of the control tasks. The authors believe that gamma coordination of CA3-CA1 activity is associated with mnemonic aspects of the spatial alternation task performance, such as memory retrieval (Montgomery and Buzsáki, 2007), while changes in theta power and coherence may be associated with others functions such as route planning (Montgomery et al., 2009).

An increase in theta coherence in the hippocampus during decision making has been shown in other studies, when animals perform a previously learned task (Schmidt et al., 2013; Belchior et al., 2014), indicating increased integration and cooperation in the hippocampus during information processing. Data obtained by intracellular recording in vivo demonstrate that the synchronization of neuronal activity in CA3 and CA1 can be up to 3 seconds to induce plasticity (Bittner et al., 2017).

Changes of theta activity in the CA1 and DG were also observed during spatial learning in the Morris water maze (Olvera-Cortés et al., 2002, 2004; Hernández-Pérez et al., 2015). In a study by Hernández-Pérez and colleagues, this problem was examined in dynamics in different layers of CA1, the CA3 pyramidal layer and DG (Hernández-Pérez et al., 2016). It was noticed that during the early stages of learning, slow frequency theta activity prevailed and it was coupled between specific layers of the hippocampal fields. However, on the last day of training, the frequency of theta oscillations was higher in all recorded layers, and their coupling was pronounced between the CA3 pyramidal layer and CA1 pyramidal layer and str. radiatum. An increase in theta coherence correlated with an improvement of task performance (Hernández-Pérez et al., 2016), once again confirming that increased synchronization between CA3-CA1 fields at the theta frequency can participate in the retrieval of spatial information.

Synchronization at theta frequency also changes during the arrival of novel information. It was shown that novel environment promoted a shift in the firing of CA1 pyramidal neurons (but not neurons of subiculum) to a later phase of the theta wave, which confirms the relationship between theta phase and CA1 plasticity induced by novelty (Lever et al., 2010). Despite the reported decrease in theta coherence along the septo-temporal axis of the hippocampus during different behaviour of the animals (running or REM sleep) (Sabolek et al., 2009; Penley et al., 2012), sensitive to animal speed (Hinman et al., 2011), the novel environment, regardless of the animal speed, led to an increase in the CA1 theta coherence a decrease in it in the DG across the long axis of the hippocampus (Penley et al., 2013). 
In support of the idea that theta rhythm and theta synchronization somehow organize the hippocampal neural network activity, there are already quite numerous studies showing that gamma oscillations and neuronal firing in the hippocampus and neocortex are phaselocked to the hippocampal theta rhythm (Fell et al., 2003b; Jones and Wilson, 2005; Siapas et al., 2005; Canolty et al., 2006; Sirota et al., 2008; Colgin et al., 2009; Nishida et al., 2014; Schomburg et al., 2014; Trimper et al., 2014; Fernández-Ruiz et al., 2017; Barth et al., 2018; Jiang et al., 2020).

\section{Conclusions}

In conclusion, we want to emphasize that the physiological role of theta and gamma coherence is different due to their different functions and mechanisms of generation. The gamma rhythm is generated by active neurons; therefore gamma coherence promotes synchronization of active neuronal ensembles. On the contrary, practically all hippocampal neurons are involved in the theta rhythm, regardless of their belonging to a particular neural ensemble. Consequently, theta coherence orchestrates the firing of principal neurons throughout the hippocampus, arranging their discharge order. From studies on the activity of a large number of neurons, we know that neurons of MEC L3 discharge first followed by the CA3 pyramidal neurons and then the CA1 pyramidal neurons (Mizuseki et al., 2009). At the same time, within the theta cycle, the neurons of the active ensemble are spiking, which is collectively reflected in the theta-gamma coupling (Buzsáki and Wang, 2012; Colgin, 2015b). How the processing of information occurs within the theta and gamma cycles? It is still unknown in modern neuroscience, but the discovery of these processes will be a breakthrough in brain research.

\section{Declarations of interest}

None

\section{Funding} 10109)

This work was supported by the Russian Science Foundation(grant number 20-71-

\section{Bibliography}

Adhikari A, Topiwala MA, Gordon JA. 2010. Synchronized activity between the ventral hippocampus and the medial prefrontal cortex during anxiety. Neuron 65:257269.

Barth AM, Domonkos A, Fernandez-Ruiz A, Freund TF, Varga V. 2018. Hippocampal Network Dynamics during Rearing Episodes. Cell Rep 23:1706-1715.

Bartos M, Vida I, Jonas P. 2007. Synaptic mechanisms of synchronized gamma oscillations in inhibitory interneuron networks. Nat Rev Neurosci 8:45-56.

Bass DI, Manns JR. 2015. Memory-enhancing amygdala stimulation elicits gamma synchrony in the hippocampus. Behav Neurosci 129:244-256.

Belchior H, Lopes-Dos-Santos V, Tort ABL, Ribeiro S. 2014. Increase in hippocampal theta oscillations during spatial decision making. Hippocampus 24:693702.

Belluscio MA, Mizuseki K, Schmidt R, Kempter R, Buzsáki G. 2012. Crossfrequency phase-phase coupling between $\theta$ and $\gamma$ oscillations in the hippocampus. $J$ Neurosci 32:423-435. 
Benchenane K, Peyrache A, Khamassi M, Tierney PL, Gioanni Y, Battaglia FP, Wiener SI. 2010. Coherent theta oscillations and reorganization of spike timing in the hippocampal- prefrontal network upon learning. Neuron 66:921-936.

Bezaire MJ, Raikov I, Burk K, Vyas D, Soltesz I. 2016. Interneuronal mechanisms of hippocampal theta oscillations in a full-scale model of the rodent CA1 circuit. elife 5.

Bezaire MJ, Soltesz I. 2013. Quantitative assessment of CA1 local circuits: knowledge base for interneuron-pyramidal cell connectivity. Hippocampus 23:751785.

Bittner KC, Milstein AD, Grienberger C, Romani S, Magee JC. 2017. Behavioral time scale synaptic plasticity underlies CA1 place fields. Science 357:1033-1036.

Börgers C, Talei Franzesi G, Lebeau FEN, Boyden ES, Kopell NJ. 2012. Minimal size of cell assemblies coordinated by gamma oscillations. PLoS Comput Biol 8:e1002362.

Borhegyi Z, Varga V, Szilágyi N, Fabo D, Freund TF. 2004. Phase segregation of medial septal GABAergic neurons during hippocampal theta activity. J Neurosci 24:8470-8479.

Borisyuk R, Hoppensteadt F. 1999. Oscillatory models of the hippocampus: a study of spatio-temporal patterns of neural activity. Biol Cybern 81:359-371.

Borisyuk R. 2002. Oscillatory activity in the neural networks of spiking elements. BioSystems 67:3-16.

Bott J-B, Muller M-A, Jackson J, Aubert J, Cassel J-C, Mathis C, Goutagny R. 2016. Spatial Reference Memory is Associated with Modulation of Theta-Gamma Coupling in the Dentate Gyrus. Cereb Cortex 26:3744-3753.

Bragin A, Jandó G, Nádasdy Z, Hetke J, Wise K, Buzsáki G. 1995. Gamma(40$100 \mathrm{~Hz}$ ) oscillation in the hippocampus of the behaving rat. J Neurosci 15:47-60.

Brazhnik ES. 2004. [Theta rhythmicity in the medial septum: entraining by the GABA-ergic neurons]. Zh Vyssh Nerv Deiat Im I P Pavlova 54:192-201.

Brunel N, Hansel D. 2006. How noise affects the synchronization properties of recurrent networks of inhibitory neurons. Neural Comput 18:1066-1110.

Brunel N. 2000. Dynamics of sparsely connected networks of excitatory and inhibitory spiking neurons. J Comput Neurosci 8:183-208.

Brun VH, Otnass MK, Molden S, Steffenach H-A, Witter MP, Moser M-B, Moser El. 2002. Place cells and place recognition maintained by direct entorhinalhippocampal circuitry. Science 296:2243-2246.

Buhl EH, Halasy K, Somogyi P. 1994. Diverse sources of hippocampal unitary inhibitory postsynaptic potentials and the number of synaptic release sites. Nature 368:823-828.

Burgess N, O'Keefe J. 2011. Models of place and grid cell firing and theta rhythmicity. Curr Opin Neurobiol 21:734-744.

Butler JL, Hay YA, Paulsen O. 2018. Comparison of three gamma oscillations in the mouse entorhinal-hippocampal system. Eur J Neurosci 48:2795-2806. 
Buzsáki G, Anastassiou CA, Koch C. 2012. The origin of extracellular fields and currents--EEG, ECoG, LFP and spikes. Nat Rev Neurosci 13:407-420.

Buzsáki G, Leung LW, Vanderwolf CH. 1983. Cellular bases of hippocampal EEG in the behaving rat. Brain Res 287:139-171.

Buzsáki G, Moser El. 2013. Memory, navigation and theta rhythm in the hippocampal-entorhinal system. Nat Neurosci 16:130-138.

Buzsáki G, Wang X-J. 2012. Mechanisms of gamma oscillations. Annu Rev Neurosci 35:203-225.

Buzsáki G. 1996. The hippocampo-neocortical dialogue. Cereb Cortex 6:81-92.

Buzsáki G. 2002. Theta oscillations in the hippocampus. Neuron 33:325-340.

Buzsáki G. 2006. Rhythms of the Brain. Oxford University Press.

Canolty RT, Edwards E, Dalal SS, Soltani M, Nagarajan SS, Kirsch HE, Berger MS, Barbaro NM, Knight RT. 2006. High gamma power is phase-locked to theta oscillations in human neocortex. Science 313:1626-1628.

Cardin JA, Carlén M, Meletis K, Knoblich U, Zhang F, Deisseroth K, Tsai L-H, Moore $\mathrm{Cl}$. 2009. Driving fast-spiking cells induces gamma rhythm and controls sensory responses. Nature 459:663-667.

Cardin JA, Carlén M, Meletis K, Knoblich U, Zhang F, Deisseroth K, Tsai L-H, Moore Cl. 2010. Targeted optogenetic stimulation and recording of neurons in vivo using cell-type-specific expression of Channelrhodopsin-2. Nat Protoc 5:247-254.

Carr MF, Karlsson MP, Frank LM. 2012. Transient slow gamma synchrony underlies hippocampal memory replay. Neuron 75:700-713.

Chang EH, Huerta PT. 2012. Neurophysiological correlates of object recognition in the dorsal subiculum. Front Behav Neurosci 6:46.

Chrobak JJ, Buzsáki G. 1998. Gamma oscillations in the entorhinal cortex of the freely behaving rat. J Neurosci 18:388-398.

Colgin LL, Denninger T, Fyhn M, Hafting T, Bonnevie T, Jensen O, Moser M-B, Moser El. 2009. Frequency of gamma oscillations routes flow of information in the hippocampus. Nature 462:353-357.

Colgin LL, Moser El. 2010. Gamma oscillations in the hippocampus. Physiology(Bethesda) 25:319-329.

Colgin LL. 2015a. Do slow and fast gamma rhythms correspond to distinct functional states in the hippocampal network? Brain Res 1621:309-315.

Colgin LL. 2015b. Theta-gamma coupling in the entorhinal-hippocampal system. Curr Opin Neurobiol 31:45-50.

Colgin LL. 2016. Rhythms of the hippocampal network. Nat Rev Neurosci 17:239-249.

Csicsvari J, Hirase H, Czurko A, Buzsáki G. 1998. Reliability and state dependence of pyramidal cell-interneuron synapses in the hippocampus: an ensemble approach in the behaving rat. Neuron 21:179-189. 
Csicsvari J, Jamieson B, Wise KD, Buzsáki G. 2003. Mechanisms of gamma oscillations in the hippocampus of the behaving rat. Neuron 37:311-322.

Cutsuridis V, Cobb S, Graham BP. 2010. Encoding and retrieval in a model of the hippocampal CA1 microcircuit. Hippocampus 20:423-446.

Cutsuridis V, Poirazi P. 2015. A computational study on how theta modulated inhibition can account for the long temporal windows in the entorhinal-hippocampal loop. Neurobiol Learn Mem 120:69-83.

Dannenberg H, Pabst M, Braganza O, Schoch S, Niediek J, Bayraktar M, Mormann F, Beck H. 2015. Synergy of direct and indirect cholinergic septohippocampal pathways coordinates firing in hippocampal networks. J Neurosci 35:8394-8410.

DeCoteau WE, Thorn C, Gibson DJ, Courtemanche R, Mitra P, Kubota Y, Graybiel AM. 2007. Learning-related coordination of striatal and hippocampal theta rhythms during acquisition of a procedural maze task. Proc Natl Acad Sci USA 104:5644-5649.

Desikan S, Koser DE, Neitz A, Monyer H. 2018. Target selectivity of septal cholinergic neurons in the medial and lateral entorhinal cortex. Proc Natl Acad Sci USA 115:E2644-E2652.

Dragoi G, Carpi D, Recce M, Csicsvari J, Buzsáki G. 1999. Interactions between hippocampus and medial septum during sharp waves and theta oscillation in the behaving rat. J Neurosci 19:6191-6199.

Durstewitz D. 2009. Implications of synaptic biophysics for recurrent network dynamics and active memory. Neural Netw 22:1189-1200.

Ecker A, Romani A, Sáray S, Káli S, Migliore M, Falck J, Lange S, Mercer A, Thomson AM, Muller E, Reimann MW, Ramaswamy S. 2020. Data-driven integration of hippocampal CA1 synaptic physiology in silico. Hippocampus 30:1129-1145.

Einevoll GT, Kayser C, Logothetis NK, Panzeri S. 2013. Modelling and analysis of local field potentials for studying the function of cortical circuits. Nat Rev Neurosci 14:770-785.

Einevoll GT, Pettersen KH, Devor A, Ulbert I, Halgren E, Dale AM. 2007. Laminar population analysis: estimating firing rates and evoked synaptic activity from multielectrode recordings in rat barrel cortex. J Neurophysiol 97:2174-2190.

Fell J, Axmacher N. 2011. The role of phase synchronization in memory processes. Nat Rev Neurosci 12:105-118.

Fell J, Fernández G, Klaver P, Elger CE, Fries P. 2003a. Is synchronized neuronal gamma activity relevant for selective attention? Brain Res Brain Res Rev 42:265-272.

Fell J, Klaver P, Elfadil H, Schaller C, Elger CE, Fernández G. 2003b. Rhinalhippocampal theta coherence during declarative memory formation: interaction with gamma synchronization? Eur J Neurosci 17:1082-1088.

Fell J, Klaver P, Lehnertz K, Grunwald T, Schaller C, Elger CE, Fernández G. 2001. Human memory formation is accompanied by rhinal-hippocampal coupling and decoupling. Nat Neurosci 4:1259-1264. 
Fernández-Ruiz A, Oliva A, Nagy GA, Maurer AP, Berényi A, Buzsáki G. 2017. Entorhinal-CA3 Dual-Input Control of Spike Timing in the Hippocampus by ThetaGamma Coupling. Neuron 93:1213-1226.e5.

Fernández-Ruiz A, Oliva A, Soula M, Rocha-Almeida F, Nagy GA, MartinVazquez G, Buzsáki G. 2021. Gamma rhythm communication between entorhinal cortex and dentate gyrus neuronal assemblies. Science 372.

Freund TF, Antal M. 1988. GABA-containing neurons in the septum control inhibitory interneurons in the hippocampus. Nature 336:170-173.

Freund TF. 1989. GABAergic septohippocampal neurons contain parvalbumin. Brain Res 478:375-381.

Fries P. 2005. A mechanism for cognitive dynamics: neuronal communication through neuronal coherence. Trends Cogn Sci(Regul Ed) 9:474-480.

Fries P. 2015. Rhythms for Cognition: Communication through Coherence. Neuron 88:220-235.

Gereke BJ, Mably AJ, Colgin LL. 2018. Experience-dependent trends in CA1 theta and slow gamma rhythms in freely behaving mice. J Neurophysiol 119:476-489.

Gilbert JR, Zarate CA. 2020. Electrophysiological biomarkers of antidepressant response to ketamine in treatment-resistant depression: Gamma power and long-term potentiation. Pharmacol Biochem Behav 189:172856.

Gonzalez-Sulser A, Parthier D, Candela A, McClure C, Pastoll H, Garden D, Sürmeli G, Nolan MF. 2014. GABAergic projections from the medial septum selectively inhibit interneurons in the medial entorhinal cortex. J Neurosci 34:16739-16743.

Hangya B, Borhegyi Z, Szilágyi N, Freund TF, Varga V. 2009. GABAergic neurons of the medial septum lead the hippocampal network during theta activity. $J$ Neurosci 29:8094-8102.

Harris KD, Csicsvari J, Hirase H, Dragoi G, Buzsáki G. 2003. Organization of cell assemblies in the hippocampus. Nature 424:552-556.

Hasselmo ME, Bodelón C, Wyble BP. 2002. A proposed function for hippocampal theta rhythm: separate phases of encoding and retrieval enhance reversal of prior learning. Neural Comput 14:793-817.

Hasselmo ME. 2005. What is the function of hippocampal theta rhythm?--Linking behavioral data to phasic properties of field potential and unit recording data. Hippocampus 15:936-949.

Hernández-Pérez JJ, Gutiérrez-Guzmán BE, López-Vázquez MÁ, Olvera-Cortés ME. 2015. Supramammillary serotonin reduction alters place learning and concomitant hippocampal, septal, and supramammillar theta activity in a Morris water maze. Front Pharmacol 6:250.

Hernández-Pérez JJ, Gutiérrez-Guzmán BE, Olvera-Cortés ME. 2016. Hippocampal strata theta oscillations change their frequency and coupling during spatial learning. Neuroscience 337:224-241.

Hinman JR, Penley SC, Long LL, Escabí MA, Chrobak JJ. 2011. Septotemporal variation in dynamics of theta: speed and habituation. J Neurophysiol 105:2675-2686. 
Hopfield JJ, Herz AV. 1995. Rapid local synchronization of action potentials: toward computation with coupled integrate-and-fire neurons. Proc Natl Acad Sci USA 92:6655-6662.

Hsiao Y-T, Zheng C, Colgin LL. 2016. Slow gamma rhythms in CA3 are entrained by slow gamma activity in the dentate gyrus. J Neurophysiol 116:25942603.

Jackson J, Amilhon B, Goutagny R, Bott J-B, Manseau F, Kortleven C, Bressler SL, Williams S. 2014. Reversal of theta rhythm flow through intact hippocampal circuits. Nat Neurosci 17:1362-1370.

Jeffery KJ. 2011. Place cells, grid cells, attractors, and remapping. Neural Plast 2011:182602.

Jiang $\mathrm{H}$, Bahramisharif A, van Gerven MAJ, Jensen O. 2020. Distinct directional couplings between slow and fast gamma power to the phase of theta oscillations in the rat hippocampus. Eur J Neurosci 51:2070-2081.

Jinno S, Kosaka T. 2006. Cellular architecture of the mouse hippocampus: a quantitative aspect of chemically defined GABAergic neurons with stereology. Neurosci Res 56:229-245.

Jones MW, Wilson MA. 2005. Phase precession of medial prefrontal cortical activity relative to the hippocampal theta rhythm. Hippocampus 15:867-873.

Joshi A, Salib M, Viney TJ, Dupret D, Somogyi P. 2017. Behavior-Dependent Activity and Synaptic Organization of Septo-hippocampal GABAergic Neurons Selectively Targeting the Hippocampal CA3 Area. Neuron 96:1342-1357.e5.

Jung MW, McNaughton BL. 1993. Spatial selectivity of unit activity in the hippocampal granular layer. Hippocampus 3:165-182.

Jutras MJ, Fries P, Buffalo EA. 2009. Gamma-band synchronization in the macaque hippocampus and memory formation. J Neurosci 29:12521-12531.

Keeley S, Fenton AA, Rinzel J. 2017. Modeling fast and slow gamma oscillations with interneurons of different subtype. J Neurophysiol 117:950-965.

Kitanishi T, Ujita S, Fallahnezhad M, Kitanishi N, Ikegaya Y, Tashiro A. 2015. Novelty-Induced Phase-Locked Firing to Slow Gamma Oscillations in the Hippocampus: Requirement of Synaptic Plasticity. Neuron 86:1265-1276.

Klausberger T, Magill PJ, Márton LF, Roberts JDB, Cobden PM, Buzsáki G, Somogyi P. 2003. Brain-state- and cell-type-specific firing of hippocampal interneurons in vivo. Nature 421:844-848.

Klausberger T. 2009. GABAergic interneurons targeting dendrites of pyramidal cells in the CA1 area of the hippocampus. Eur J Neurosci 30:947-957.

Kocsis B, Bragin A, Buzsáki G. 1999. Interdependence of multiple theta generators in the hippocampus: a partial coherence analysis. J Neurosci 19:62006212.

Kocsis B, Vertes RP. 1994. Characterization of neurons of the supramammillary nucleus and mammillary body that discharge rhythmically with the hippocampal theta rhythm in the rat. J Neurosci 14:7040-7052. 
Larkin MC, Lykken C, Tye LD, Wickelgren JG, Frank LM. 2014. Hippocampal output area CA1 broadcasts a generalized novelty signal during an object-place recognition task. Hippocampus 24:773-783.

Lasztóczi B, Klausberger T. 2017. Distinct gamma oscillations in the distal dendritic fields of the dentate gyrus and the CA1 area of mouse hippocampus. Brain Struct Funct 222:3355-3365.

Lever C, Burton S, Jeewajee A, Wills TJ, Cacucci F, Burgess N, O'Keefe J. 2010. Environmental novelty elicits a later theta phase of firing in CA1 but not subiculum. Hippocampus 20:229-234.

López-Madrona VJ, Pérez-Montoyo E, Álvarez-Salvado E, Moratal D, Herreras O, Pereda E, Mirasso CR, Canals S. 2020. Different theta frameworks coexist in the rat hippocampus and are coordinated during memory-guided and novelty tasks. elife 9.

Lubenov EV, Siapas AG. 2009. Hippocampal theta oscillations are travelling waves. Nature 459:534-539.

Lundqvist M, Rose J, Herman P, Brincat SL, Buschman TJ, Miller EK. 2016. Gamma and beta bursts underlie working memory. Neuron 90:152-164.

Manns JR, Zilli EA, Ong KC, Hasselmo ME, Eichenbaum H. 2007. Hippocampal CA1 spiking during encoding and retrieval: relation to theta phase. Neurobiol Learn Mem 87:9-20.

Middleton SJ, McHugh TJ. 2016. Silencing CA3 disrupts temporal coding in the CA1 ensemble. Nat Neurosci 19:945-951.

Migliore R, Lupascu CA, Bologna LL, Romani A, Courcol J-D, Antonel S, Van Geit WAH, Thomson AM, Mercer A, Lange S, Falck J, Rössert CA, Shi Y, Hagens O, Pezzoli M, Freund TF, Kali S, Muller EB, Schürmann F, Markram H, Migliore M. 2018. The physiological variability of channel density in hippocampal CA1 pyramidal cells and interneurons explored using a unified data-driven modeling workflow. PLoS Comput Biol 14:e1006423.

Mizuseki K, Sirota A, Pastalkova E, Buzsáki G. 2009. Theta oscillations provide temporal windows for local circuit computation in the entorhinal-hippocampal loop. Neuron 64:267-280.

Montefusco-Siegmund R, Leonard TK, Hoffman KL. 2017. Hippocampal gammaband Synchrony and pupillary responses index memory during visual search. Hippocampus 27:425-434.

Montgomery SM, Betancur MI, Buzsáki G. 2009. Behavior-dependent coordination of multiple theta dipoles in the hippocampus. J Neurosci 29:1381-1394.

Montgomery SM, Buzsáki G. 2007. Gamma oscillations dynamically couple hippocampal $\mathrm{CA} 3$ and $\mathrm{CA} 1$ regions during memory task performance. Proc Natl Acad Sci USA 104:14495-14500.

Montoya CP, Sainsbury RS. 1985. The effects of entorhinal cortex lesions on type 1 and type 2 theta. Physiol Behav 35:121-126.

Mysin IE, Kitchigina VF, Kazanovich Y. 2015. Modeling synchronous theta activity in the medial septum: key role of local communications between different cell populations. J Comput Neurosci 39:1-16. 
Nandi B, Swiatek P, Kocsis B, Ding M. 2019. Inferring the direction of rhythmic neural transmission via inter-regional phase-amplitude coupling(ir-PAC). Sci Rep 9:6933.

Nishida H, Takahashi M, Lauwereyns J. 2014. Within-session dynamics of thetagamma coupling and high-frequency oscillations during spatial alternation in rat hippocampal area CA1. Cogn Neurodyn 8:363-372.

Ohara S, Sato S, Tsutsui K-I, Witter MP, lijima T. 2013. Organization of multisynaptic inputs to the dorsal and ventral dentate gyrus: retrograde trans-synaptic tracing with rabies virus vector in the rat. PLoS ONE 8:e78928.

Olvera-Cortés E, Cervantes M, González-Burgos I. 2002. Place-learning, but not cue-learning training, modifies the hippocampal theta rhythm in rats. Brain Res Bull 58:261-270.

Olvera-Cortés E, Guevara MA, González-Burgos I. 2004. Increase of the hippocampal theta activity in the Morris water maze reflects learning rather than motor activity. Brain Res Bull 62:379-384.

Patel J, Fujisawa S, Berényi A, Royer S, Buzsáki G. 2012. Traveling theta waves along the entire septotemporal axis of the hippocampus. Neuron 75:410-417.

Penley SC, Hinman JR, Long LL, Markus EJ, Escabí MA, Chrobak JJ. 2013. Novel space alters theta and gamma synchrony across the longitudinal axis of the hippocampus. Front Syst Neurosci 7:20.

Penley SC, Hinman JR, Sabolek HR, Escabí MA, Markus EJ, Chrobak JJ. 2012. Theta and gamma coherence across the septotemporal axis during distinct behavioral states. Hippocampus 22:1164-1175.

Petsche H, Stumpf C. 1960. Topographic and toposcopic study of origin and spread of the regular synchronized arousal pattern in the rabbit. Electroencephalogr Clin Neurophysiol 12:589-600.

Pettersen KH, Hagen E, Einevoll GT. 2008. Estimation of population firing rates and current source densities from laminar electrode recordings. J Comput Neurosci 24:291-313.

Pike FG, Goddard RS, Suckling JM, Ganter P, Kasthuri N, Paulsen O. 2000. Distinct frequency preferences of different types of rat hippocampal neurones in response to oscillatory input currents. J Physiol(Lond) 529 Pt 1:205-213.

de la Prida LM, Huberfeld G. 2019. Inhibition and oscillations in the human brain tissue in vitro. Neurobiol Dis 125:198-210.

Robinson J, Manseau F, Ducharme G, Amilhon B, Vigneault E, El Mestikawy S, Williams S. 2016. Optogenetic activation of septal glutamatergic neurons drive hippocampal theta rhythms. J Neurosci 36:3016-3023.

Royer S, Sirota A, Patel J, Buzsáki G. 2010. Distinct representations and theta dynamics in dorsal and ventral hippocampus. J Neurosci 30:1777-1787.

Sabolek HR, Penley SC, Hinman JR, Bunce JG, Markus EJ, Escabi M, Chrobak JJ. 2009. Theta and gamma coherence along the septotemporal axis of the hippocampus. J Neurophysiol 101:1192-1200. 
Salib M, Joshi A, Katona L, Howarth M, Micklem BR, Somogyi P, Viney TJ. 2019. GABAergic Medial Septal Neurons with Low-Rhythmic Firing Innervating the Dentate Gyrus and Hippocampal Area CA3. J Neurosci 39:4527-4549.

Saraga F, Ng L, Skinner FK. 2006. Distal gap junctions and active dendrites can tune network dynamics. J Neurophysiol 95:1669-1682.

Schmidt B, Hinman JR, Jacobson TK, Szkudlarek E, Argraves M, Escabí MA, Markus EJ. 2013. Dissociation between dorsal and ventral hippocampal theta oscillations during decision-making. J Neurosci 33:6212-6224.

Schneider CJ, Bezaire M, Soltesz I. 2012. Toward a full-scale computational model of the rat dentate gyrus. Front Neural Circuits 6:83.

Schneider J, Lewen A, Ta T-T, Galow LV, Isola R, Papageorgiou IE, Kann O. 2015. A reliable model for gamma oscillations in hippocampal tissue. J Neurosci Res 93:1067-1078.

Schomburg EW, Fernández-Ruiz A, Mizuseki K, Berényi A, Anastassiou CA, Koch C, Buzsáki G. 2014. Theta phase segregation of input-specific gamma patterns in entorhinal-hippocampal networks. Neuron 84:470-485.

Seidenbecher T, Laxmi TR, Stork O, Pape H-C. 2003. Amygdalar and hippocampal theta rhythm synchronization during fear memory retrieval. Science 301:846-850.

Senior TJ, Huxter JR, Allen K, O’Neill J, Csicsvari J. 2008. Gamma oscillatory firing reveals distinct populations of pyramidal cells in the CA1 region of the hippocampus. J Neurosci 28:2274-2286.

Sheremet A, Zhou Y, Qin Y, Kennedy JP, Lovett SD, Maurer AP. 2020. An investigation into the nonlinear coupling between CA1 layers and the dentate gyrus. Behav Neurosci 134:491-515.

Siapas AG, Lubenov EV, Wilson MA. 2005. Prefrontal phase locking to hippocampal theta oscillations. Neuron 46:141-151.

Sirota A, Montgomery S, Fujisawa S, Isomura Y, Zugaro M, Buzsáki G. 2008. Entrainment of neocortical neurons and gamma oscillations by the hippocampal theta rhythm. Neuron 60:683-697.

Skaggs WE, McNaughton BL, Wilson MA, Barnes CA. 1996. Theta phase precession in hippocampal neuronal populations and the compression of temporal sequences. Hippocampus 6:149-172.

Soltesz I, Deschênes M. 1993. Low- and high-frequency membrane potential oscillations during theta activity in CA1 and CA3 pyramidal neurons of the rat hippocampus under ketamine-xylazine anesthesia. J Neurophysiol 70:97-116.

Somogyi P, Katona L, Klausberger T, Lasztóczi B, Viney TJ. 2014. Temporal redistribution of inhibition over neuronal subcellular domains underlies statedependent rhythmic change of excitability in the hippocampus. Philos Trans R Soc Lond B Biol Sci 369:20120518.

Steffenach H-A, Sloviter RS, Moser El, Moser M-B. 2002. Impaired retention of spatial memory after transection of longitudinally oriented axons of hippocampal CA3 pyramidal cells. Proc Natl Acad Sci USA 99:3194-3198. 
Stepan J, Dine J, Fenzl T, Polta SA, von Wolff G, Wotjak CT, Eder M. 2012. Entorhinal theta-frequency input to the dentate gyrus trisynaptically evokes hippocampal CA1 LTP. Front Neural Circuits 6:64.

Stewart M, Fox SE. 1990. Do septal neurons pace the hippocampal theta rhythm? Trends Neurosci 13:163-168.

Sutherland RJ, Whishaw IQ, Kolb B. 1983. A behavioural analysis of spatial localization following electrolytic, kainate- or colchicine-induced damage to the hippocampal formation in the rat. Behav Brain Res 7:133-153.

Tort ABL, Komorowski RW, Manns JR, Kopell NJ, Eichenbaum H. 2009. Thetagamma coupling increases during the learning of item-context associations. Proc Natl Acad Sci USA 106:20942-20947.

Traub RD, Whittington MA, Colling SB, Buzsáki G, Jefferys JG. 1996. Analysis of gamma rhythms in the rat hippocampus in vitro and in vivo. J Physiol(Lond) 493( Pt 2):471-484.

Trimper JB, Galloway CR, Jones AC, Mandi K, Manns JR. 2017. Gamma oscillations in rat hippocampal subregions dentate gyrus, CA3, CA1, and subiculum underlie associative memory encoding. Cell Rep 21:2419-2432.

Trimper JB, Stefanescu RA, Manns JR. 2014. Recognition memory and thetagamma interactions in the hippocampus. Hippocampus 24:341-353.

Ujfalussy B, Kiss T. 2006. How do glutamatergic and GABAergic cells contribute to synchronization in the medial septum? J Comput Neurosci 21:343-357.

Vertes RP, Kocsis B. 1997. Brainstem-diencephalo-septohippocampal systems controlling the theta rhythm of the hippocampus. Neuroscience 81:893-926.

Viney TJ, Salib M, Joshi A, Unal G, Berry N, Somogyi P. 2018. Shared rhythmic subcortical GABAergic input to the entorhinal cortex and presubiculum. elife 7.

Vinogradova OS. 1995. Expression, control, and probable functional significance of the neuronal theta-rhythm. Prog Neurobiol 45:523-583.

Vinogradova OS. 2001. Hippocampus as comparator: role of the two input and two output systems of the hippocampus in selection and registration of information. Hippocampus 11:578-598.

Wang WT, Wan YH, Zhu JL, Lei GS, Wang YY, Zhang P, Hu SJ. 2006. Thetafrequency membrane resonance and its ionic mechanisms in rat subicular pyramidal neurons. Neuroscience 140:45-55.

Wang X-J. 2010. Neurophysiological and computational principles of cortical rhythms in cognition. Physiol Rev 90:1195-1268.

Wang XJ, Buzsáki G. 1996. Gamma oscillation by synaptic inhibition in a hippocampal interneuronal network model. J Neurosci 16:6402-6413.

Womelsdorf T, Schoffelen J-M, Oostenveld R, Singer W, Desimone R, Engel AK, Fries P. 2007. Modulation of neuronal interactions through neuronal synchronization. Science 316:1609-1612.

Yamamoto J, Tonegawa S. 2017. Direct medial entorhinal cortex input to hippocampal CA1 is crucial for extended quiet awake replay. Neuron 96:217-227.e4. 
Ylinen A, Soltész I, Bragin A, Penttonen M, Sik A, Buzsáki G. 1995. Intracellular correlates of hippocampal theta rhythm in identified pyramidal cells, granule cells, and basket cells. Hippocampus 5:78-90.

Yoshida K, Oka H. 1995. Topographical projections from the medial septumdiagonal band complex to the hippocampus: a retrograde tracing study with multiple fluorescent dyes in rats. Neurosci Res 21:199-209.

Zhang $\mathrm{H}$, Jacobs J. 2015. Traveling theta waves in the human hippocampus. J Neurosci 35:12477-12487.

Zheng C, Bieri KW, Hwaun E, Colgin LL. 2016. Fast Gamma Rhythms in the Hippocampus Promote Encoding of Novel Object-Place Pairings. Eneuro 3.

Zheng C, Bieri KW, Trettel SG, Colgin LL. 2015. The relationship between gamma frequency and running speed differs for slow and fast gamma rhythms in freely behaving rats. Hippocampus 25:924-938. 